\title{
IRON: uma abordagem inteligente para roteamento em redes ópticas elásticas
}

\author{
Neclyeux Monteiro ${ }^{1}$ e André Soares ${ }^{1}$ \\ ${ }^{1}$ Departamento de Computação - Universidade Federal do Piauí (UFPI) \\ Teresina - PI - Brasil \\ neclyeuxsousa@gmail.com
}

\begin{abstract}
Elastic optical networks comprise a network infrastructure capable of supporting the high demand for Internet data traffic. The Routing, Modulation Level and Spectrum Assignment (RMLSA) is one of the main problems that must be solved to ensure the successful performance of this type of network. This paper focuses on a new solution to the routing problem. The new solution uses a Multi Layer Perceptron neural network and is called Intelligent Routing for Optical Networks (IRON). The solution is compared with the Complete Sharing algorithm in two different scenarios using the Cost239 and Pan-European topologies. The IRON algorithm obtained a reduction in bandwidth blocking probability of approximately $28.59 \%$ and $21.83 \%$ compared to Complete Sharing in the Cost 239 and Pan-European topologies respectively.
\end{abstract}

Resumo. As redes ópticas elásticas compõem uma infraestrutura de rede capaz de suportar a grande demanda de tráfego de dados da Internet. Um dos principais problemas que devem ser solucionados para garantir o bom funcionamento deste tipo de rede é o chamado Routing, Modulation Level and Spectrum Assignment (RMLSA). Este artigo foca em uma nova solução para o problema de roteamento. A nova solução utiliza uma rede neural Multi Layer Perceptron e é chamada de Intelligent Routing for Optical Networks (IRON). A solução é comparada com o algoritmo Complete Sharing em dois cenários diferentes utilizando as topologias Cost239 e Pan-European. O algoritmo IRON obteve uma redução na probabilidade de bloqueio de banda de aproximadamente $28,59 \%$ e 21,83\% em relação ao Complete Sharing nas topologias Cost239e Pan-European respectivamente.

\section{Introdução}

Devido ao constante crescimento no tráfego de dados da Internet, é necessária uma evolução da infraestrutura de rede capaz de suportar essa demanda. Nesse sentido, as redes ópticas elásticas surgem como proposta para melhorar o uso dos recursos ópticos e atender os tráfegos de dados das aplicações que demandam cada vez mais uma maior e melhor largura de banda [Wu et al. 2017]. Uma rede óptica elástica, possui a capacidade de alocar espectro de maneira flexível, atendendo as diferentes larguras de banda com uma quantidade diferente de slots de frequência óptica [Chatterjee et al. 2015].

Um desafio encontrado nas redes ópticas elásticas é utilizar os recursos espectrais de tal maneira que garanta um desempenho eficiente, buscando reduzir ao máximo os 
bloqueios de requisições de circuitos ópticos. Dentre os problemas encontrados na literatura para este tipo de rede destaca-se o problema de roteamento, seleção de formato de modulação e alocação de espectro (Routing, Modulation Level, and Spectrum Assignment - RMLSA) [Christodoulopoulos et al. 2011]. Para buscar um uso eficiente dos recursos é necessário resolver o problema RMLSA, que consiste basicamente de três subproblemas: 1) definir uma rota para um par de nós; 2) selecionar um formato de modulação; e 3) definir uma faixa do espectro livre para estabelecimento do circuito óptico.

Este trabalho tem foco no subproblema de roteamento. De maneira geral, destaca-se três diferentes abordagens de roteamento encontradas na literatura: 1) Roteamento Fixo; 2) Roteamento Fixo Alternativo; e 3) Roteamento Adaptativo [Chatterjee et al. 2015].

Este trabalho propõe a utilização de uma técnica de Machine Learning (ML) para tratar do problema de roteamento de maneira fixa alternativa. A técnica escolhida foi a rede neural Multi Layer Perceptron (MLP), devido a sua capacidade de aprendizado através da análise das características da rede e da requisição no momento da chegada de uma requisição para o estabelecimento de um circuito.

O algoritmo proposto é chamado de Intelligent Routing for Optical Networks (IRON). A proposta consiste em treinar uma rede neural MLP para analisar as característica da requisição e do estado atual dos enlaces que compõem as $k$ rotas candidatas. A proposta é comparada com o algoritmo encontrado na literatura chamado de Complete Sharing [Wang and Mukherjee 2014]. Os resultados mostram que a escolha da rota realizada pela rede neural se mostra mais eficiente para redução da probabilidade de bloqueio geral da rede.

As principais contribuições deste artigo são: i) Apresentar uma solução de roteamento fixo-alternativo para redes ópticas elásticas utilizando ML; ii) Apresentar evidencias de que uma dada heurística já conhecida na área de redes ópticas elásticas pode ser utilizada para criar uma base de dados de aprendizado, obtendo uma nova solução que utiliza ML que alcança resultados melhores do que a heurística original.

As demais seções deste artigo estão organizadas da seguinte forma. Na Seção 2 são apresentados os principais conceitos da arquitetura utilizada pela rede óptica elástica, bem como é descrito o problema RMLSA. A Seção 3 discute os trabalhos relacionados ao problema de roteamento que utilizam técnicas de Machine Learning. A Seção 4 apresenta os conceitos das redes neurais artificiais e descreve o tipo de rede neural utilizada neste trabalho. O algoritmo proposto é apresentado na Seção 5. A Seção 6 apresenta um estudo de avaliação de desempenho da proposta. Por fim, as conclusões e trabalhos futuros são apresentadas na Seção 7.

\section{Redes Ópticas Elásticas}

A divisão do espectro óptico em vários slots de frequência, possibilitou o surgimento de um novo tipo de rede óptica de transporte chamada de rede óptica elástica, proposta em [Jinno et al. 2009]. Nesse tipo de rede, para haver comunicação, é necessário o estabelecimento de um circuito óptico entre um par de nós origem e destino. Cada circuito é composto por um ou mais slots, isso depende da largura de banda requisitada pelo circuito. A Figura 1 apresenta um exemplo de divisão do espectro óptico em slots de frequência. 


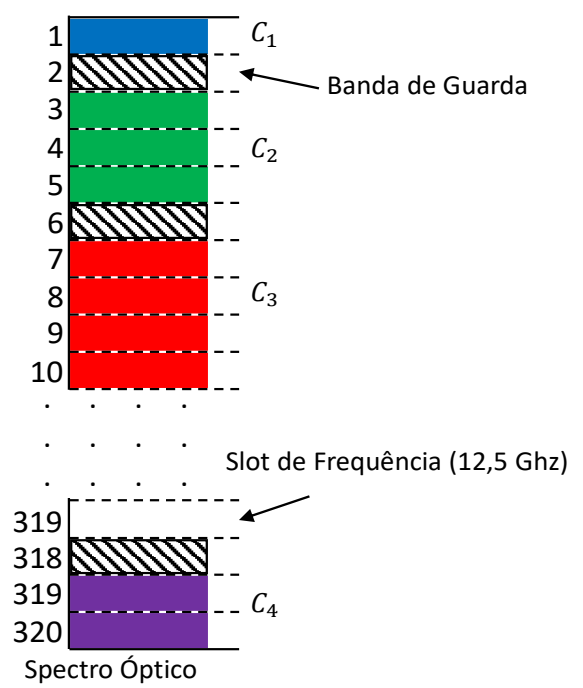

Figura 1. Exemplo de divisão do espectro óptico em slots de frequência.

Ao observar a Figura 1, os slots brancos representam os slots livres enquanto os slots coloridos representam circuitos ativos com diferentes larguras de banda. Por exemplo, o circuito $c_{1}$ aloca apenas um slot de frequência, enquanto o circuito $c_{3}$ aloca quatro slots de frequência. Ilustra-se ainda na Figura 1 a banda de guarda que é usada entre os circuitos adjacentes com a finalidade de reduzir a interferência entre eles [Zhao et al. 2015].

Para assegurar essa flexibilidade na alocação espectral, as redes ópticas utilizam duas tecnologias: Transponders com largura de banda variável (Bandwidth Variable Transponders - BVT) e comutador óptico com largura de banda variável (Bandwidth Variable Wavelength Cross-Connects - BV-WXC). Os BVTs são utilizados para garantir a flexibilidade e o ajuste da largura de banda entre os nós origem e destino da requisição. Os BV-WXCs permitem a comutação óptica de circuitos [Chatterjee et al. 2015].

Durante a transmissão do sinal óptico pelos enlaces, quanto maior a distância percorrida na fibra, maior será a perda de potência do sinal óptico. Em contrapartida, a potência do ruído aumenta gradativamente, podendo deixar o sinal óptico indecifrável no receptor. Essa relação é chamada de relação sinal-ruído óptica (Optical Signal to Noise Ratio - OSNR). Isso pode ocorrer de tal maneira que seja necessário amplificar o sinal óptico para reestabelecer a potência e o sinal ser detectado no seu destino. Esta amplificação é realizada pelo equipamento amplificador (Erbium Doped Fiber Amplifier - EDFA). No entanto, no momento desta amplificação, os EDFAs acabam introduzindo o ruído da emissão espontânea amplificada (Amplified Spontaneous Emission - ASE) [Saradhi and Subramaniam 2009]. Além disso, quando o sinal se propaga pela fibra, sofre ainda ruídos causados pelos efeitos não lineares (Nonlinear Impairments NLIs).

O modelo de camada física apresentado em [Johannisson and Agrell 2014, Yan et al. 2015] foi adotado neste trabalho para medir os impactos causados pelos ruídos ASE e NLIs na OSNR. A OSNR é utilizada para a avaliação da qualidade de transmissão (Quality of Transmission - QoT) dos circuitos ópticos. Caso o valor de OSNR do circuito não seja maior ou igual um limiar definido, o estabelecimento do circuito pode acabar 
sendo bloqueado por falta de QoT. O bloqueio por falta de QoT pode acontecer de duas formas: 1) QoT inadequada para o novo circuito (QoTN) ou 2) QoT inadequada para um ou mais circuitos já ativos na rede (QoTO). O QoTN ocorre quando o circuito não atinge o nível de QoT necessário para seu estabelecimento. A QoTO ocorre quando o estabelecimento do desse circuito afeta demasiadamente de forma negativa a QoT dos circuitos já estabelecidos na rede [Fontinele et al. 2017].

Para um uso eficiente dos recursos das redes ópticas é necessário solucionar o problema RMLSA. Como mencionado, na primeira etapa deste problema é necessário escolher uma rota entre o par de nós origem e destino da requisição. A Figura 2, apresenta uma topologia exemplo e mostra três possíveis escolhas de rota para uma requisição com origem no nó 1 e destino no nó 4 . As três opções possíveis de rota são: 1) 1-2-3-4;2) $1-2-5-4$ e 3) 1-6-5-4.

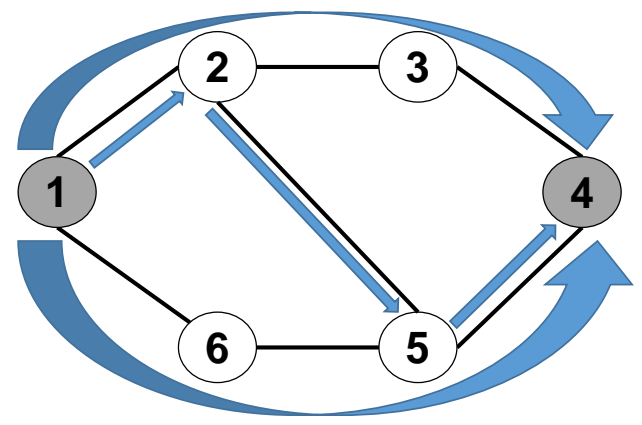

Figura 2. Topologia exemplo e problema de roteamento entre os nós 1 e 4.

Com a rota selecionada, o próximo passo é a escolha de um formato de modulação adequado. $\mathrm{O}$ formato de modulação em conjunto com a taxa de transmissão solicitada determinam a quantidade de slots necessários para atendimento do circuito. A escolha do formato de modulação está relacionada ao tamanho da rota escolhida no passo anterior. Modulações com uma maior eficiência espectral sofrem mais ruídos vindos da camada física e por isso possuem um alcance menor. Dessa forma, modulações com uma eficiência espectral menor tendem a ser mais adequadas para rotas maiores.

Por último, é necessária a escolha da faixa espectral para alocar o circuito óptico. Nessa etapa deve-se atender a duas restrições: as restrições de continuidade e contiguidade. A restrição de continuidade determina que o sinal óptico mantenha-se no mesmo intervalo espectral em todos os enlaces da rota, ou seja, a mesma faixa de slots deve estar livre em todos os enlaces para atender a requisição. Na restrição de contiguidade, para requisições que exigem 2 ou mais slots, estes slots devem ser alocados de forma adjacente, contíguos entre si.

A medida que os circuitos são estabelecidos e suas conexões finalizadas, a rede tende a apresentar problemas como fragmentação, aumentando assim a probabilidade das restrições de continuidade e contiguidade não serem atendidas, causando bloqueios de futuras requisições devido a fragmentação do espectro. Quando a rede possui slots suficientes para atender a requisição mas estes slots não respeitam as restrições de continuidade e contiguidade, ocorre o bloqueio por Fragmentação. Quando a rede não possui slots suficientes para atender a requisição, ocorre bloqueio por Ausência de Espectro Livre (AEL). 


\section{Trabalhos Relacionados}

Um dos problemas fundamentais nas redes ópticas é o problema de roteamento. O problema de roteamento consiste basicamente em encontrar uma rota entre um par de nós origem e destino para alocar um circuito. No entanto, devido ao crescimento e complexidade das redes ópticas, o roteamento de menor caminho pode acabar não sendo mais uma boa opção e resultar em um alto bloqueio de requisições.

Nesse contexto, alguns trabalhos encontrados na literatura utilizam técnicas de Machine Learning para resolver o problema de roteamento buscando aumentar a capacidade de atendimento das redes, reduzindo assim a probabilidade de bloqueio.

Em [Levesque and Elbiaze 2009] os autores propõem um algoritmo chamado de Graphical Probabilistic Routing Model (GPRM) que seleciona os enlaces menos utilizados e vai construindo a rota enlace por enlace, utilizando Bayesian Network em um cenário de Optical Burst Switching (OBS). Os resultados mostram que o algoritmo GPRM é mais eficiente para reduzir a taxa de rupturas da rede quando comparado com propostas não adaptativas de roteamento.

Em [Troia et al. 2018] o problema de roteamento é modelado com um problema de classificação em um cenário de redes definidas por software. Os autores criam um modelo chamado de Machine Learning Routing Computation (MLRC) que captura o estado atual do tráfego da rede e classifica esse tráfego para definir uma rota ótima para o estado atual da rede.

Em [Zhong et al. 2019] os autores apresentam um cenário de redes multi-domínio no qual o acesso de uma rede é protegido contra acesso externo por questões de segurança e privacidade. Os autores propõem então um algoritmo para roteamento inter-domínios, usando uma análise de histórico de dados para treinar o modelo de aprendizagem profunda para retornar rotas inter-domínios viáveis quando solicitado.

Alguns aspectos dos trabalhos listados se diferem do trabalho deste artigo, vejamos: i) Em [Levesque and Elbiaze 2009] o roteamento utilizado é do tipo adaptativo em uma rede de comutação de rajadas ópticas (OBS) ; ii) Em [Troia et al. 2018] os autores estudam as redes definidas por software. Além disso, os autores não detalham suficientemente a estratégia utilizada, o que impede a implementação para fins comparativos; e iii) Em [Zhong et al. 2019] o algoritmo foi proposto para um cenário de redes multidomínios.

Diante do exposto, bem como o número reduzido de trabalhos encontrados na literatura que utilizam Machine Learning para o problema de roteamento, este trabalho propõe a utilização da técnica descrita na Seção 4 para roteamento do tipo fixo-alternativo em um cenário de redes ópticas elásticas. O algoritmo proposto é apresentado na Seção 5 .

\section{Redes Neurais Artificiais}

Redes neurais artificiais vem ganhando cada vez mais espaço na literatura devido a sua capacidade de autoaprendizagem e aplicabilidade em diversos problemas [Khokhar et al. 2015], tais como processar sinais de áudio, fala, conteúdos visuais (imagens e vídeos) e textuais. No contexto das redes ópticas elásticas, diferentes autores vem propondo aplicações das redes neurais nos diversos problemas encontrados nesse 
tipo de rede, tais como: roteamento, desfragmentação, realocação de circuitos, escolha do tamanho da banda de guarda e etc [Troia et al. 2018, Trindade and da Fonseca 2021, Rodrigues et al. 2020].

Os métodos convencionais de aprendizagem de máquina dependem muito das características extraídas, que necessitam de um esforço significativo para encontrar as características mais adequadas [Mohan et al. 2017]. Nos modelos de aprendizagem profunda é necessário uma grande quantidade de dados de entrada para realizar o processo de aprendizagem. Esses modelos recebem os dados de entrada e realizam a extração, seleção e classificação das características. Neste trabalho foi utilizada a rede neural Multi Layer Perceptrons (MLPs).

Neste tipo de rede neural, os neurônios são interconectados formando várias camadas, nas quais é feito o mapeamento linear das características usadas como entrada até a saída desejada [Gardner and Dorling 1998]. A saída de um neurônio na camada $x$ é usada como entrada para o neurônio da camada $x+1$, assim o processamento dos dados é feito sempre da camada de entrada para a camada de saída. Redes neurais desse tipo são chamadas de redes neurais feed-foward.

A rede neural MLP possui a capacidade de aprender através da realização de um treinamento. Esse treinamento é feito com um conjunto de dados que consistem em características, usadas como entrada para a rede neural, bem como uma última característica que consiste na saída desejada. No contexto deste trabalho, para extração e composição da base de dados usada para treinamento da rede neural foi realizada uma simulação prévia com um algoritmo base para extração destas características. A Figura 3 demonstra esse procedimento.

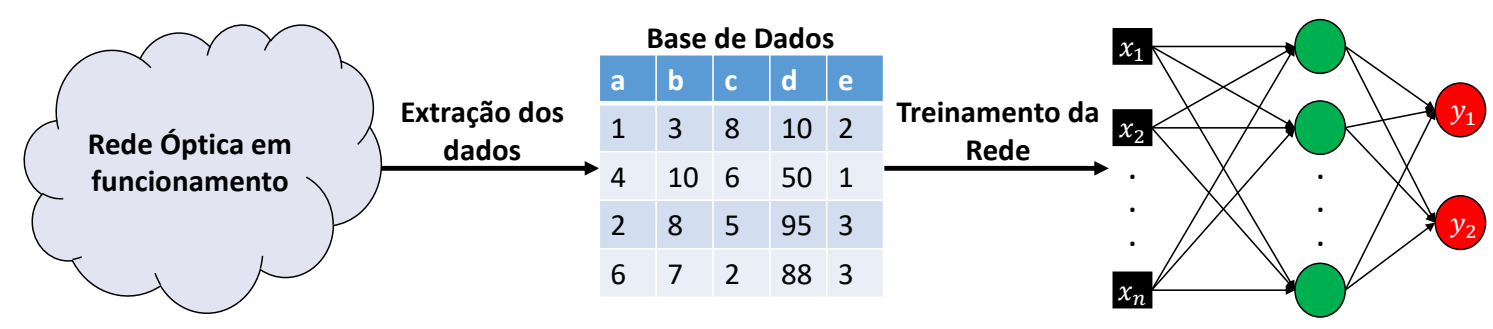

Figura 3. Fluxograma de extração dos dados e treinamento da Rede Neural.

A base de dados contendo as características (entradas e saídas) é apresentada repetidamente para a rede MLP, e os pesos são ajustados durante o treinamento de forma que o mapeamento correto entre a entrada e a saída desejada ocorra. No entanto, durante o treinamento pode ocorrer da saída não ser a esperada, gerando assim um sinal de erro que nada mais é do que a diferença entre a saída desejada e a saída obtida. A rede neural ajusta os pesos baseado nesse erro a fim de minimizar o erro geral da rede MLP. O algoritmo utilizado neste trabalho para cálculo do erro e ajuste dos pesos foi o backprogation [Hecht-Nielsen 1992].

Durante o treinamento da rede MLP, vários hiperparâmetros precisam ser configurados, a fim de obter um melhor desempenho da rede. Dentre esses hiperparâmetros que precisam ser otimizados podemos citar: Quantidade de épocas, de neurônios, de camadas ocultas, valor da taxa de aprendizagem e etc. Devido a quantidade de parâmetros que 
precisam ser ajustados e otimizados, as redes MLP estão sujeitas a diversos problemas como overfitting [Hawkins 2004] e oscilações no ótimo [Wen Jin et al. 2000].

Umas das técnicas utilizadas para evitar o overfitting é a chamada técnica da parada precoce, ou Early stop. Tal técnica permite especificar um número $x$ de épocas e parar o treinamento da rede caso o desempenho não venha a melhorar após passadas essas $x$ épocas. Para evitar o problema de oscilações ao ótimo, utiliza-se durante o treinamento uma taxa de aprendizagem dinâmica. Dessa forma, a taxa de aprendizagem é diminuída conforme a rede não apresente uma melhora no treinamento, buscando assim uma aproximação ao valor ótimo.

Diante do exposto, a rede MLP produzida neste trabalho possui quatro camadas completamente conectadas, possuindo respectivamente 128, 64, 32 e 3 neurônios em cada camada. A quantidade de neurônios e camadas foram definidos empiricamente, com base no conhecimento do especialista e estudos realizados no âmbito das redes neurais. Nas três primeiras camadas a função de ativação utilizada foi a ReLU (Rectified Linear Units) [LeCun et al. 2015] e na última camada (camada de saída) a função utilizada foi a softmax [Wang and Chen 2019], que fornece a probabilidade dos dados de entrada pertencerem a uma classe correspondente na saída.

\section{Intelligent Routing for Optical Networks - IRON}

O algoritmo proposto para escolha da rota neste trabalho utiliza a técnica descrita na Seção 4. O algoritmo é chamado de IRON (Intelligent Routing for Optical Networks). Para compor a base de dados, foi utilizado o algoritmo Complete Sharing, conforme demonstrado na Figura 3. O Complete Sharing é um algoritmo RMLSA integrado proposto para lidar com a fragmentação do espectro. Este algoritmo utiliza um roteamento com $k$ rotas candidatas e seleciona aquela em que é possível estabelecer o circuito em uma faixa de slots mais próxima do início do espectro. Ele utiliza o First Fit para realizar a alocação de espectro.

A base de dados utilizada possui registros que representam características da requisição e das rotas candidatas a serem escolhidas. As características escolhidas para compor a base de dados de treinamento foram as seguintes: a) Nó de origem da requisição; b) Nó de destino da requisição; c) Número de enlaces da rota candidata 1; d) Número de slots livres da rota candidata 1; e) Número de enlaces da rota candidata 2; f) Número de slots livres da rota candidata 2; g) Número de enlaces da rota candidata 3; h) Número de slots livres da rota candidata 3; e por fim a variável de saída i) Número da rota escolhida. A Tabela 1 mostra alguns exemplos de registros que estão contidos na base de dados utilizada durante o treinamento da rede.

Tabela 1. Exemplo de alguns registros contidas na base de dados extraída do Complete Sharing.

\begin{tabular}{c|c|c|c|c|c|c|c|c}
\hline $\mathbf{a}$ & $\mathbf{b}$ & $\mathbf{c}$ & $\mathbf{d}$ & $\mathbf{e}$ & $\mathbf{f}$ & $\mathbf{g}$ & $\mathbf{h}$ & $\mathbf{i}$ \\
\hline 10 & 1 & 2 & 536 & 2 & 549 & 3 & 829 & 1 \\
\hline 8 & 2 & 1 & 273 & 2 & 561 & 3 & 810 & 2 \\
\hline 6 & 2 & 2 & 540 & 2 & 546 & 2 & 509 & 1 \\
\hline 3 & 7 & 2 & 529 & 2 & 502 & 2 & 539 & 3 \\
\hline
\end{tabular}


Vale ressaltar que durante a fase de extração das características para compor a base de dados, requisições que sofreram bloqueio foram descartadas da base de dados. Isto foi feito para que a rede neural apenas aprendesse os padrões e comportamentos nos quais foram feitos escolhas de rotas em que a requisição obtivesse sucesso. A Figura 4 apresenta o fluxograma para aplicação da proposta desenvolvida.

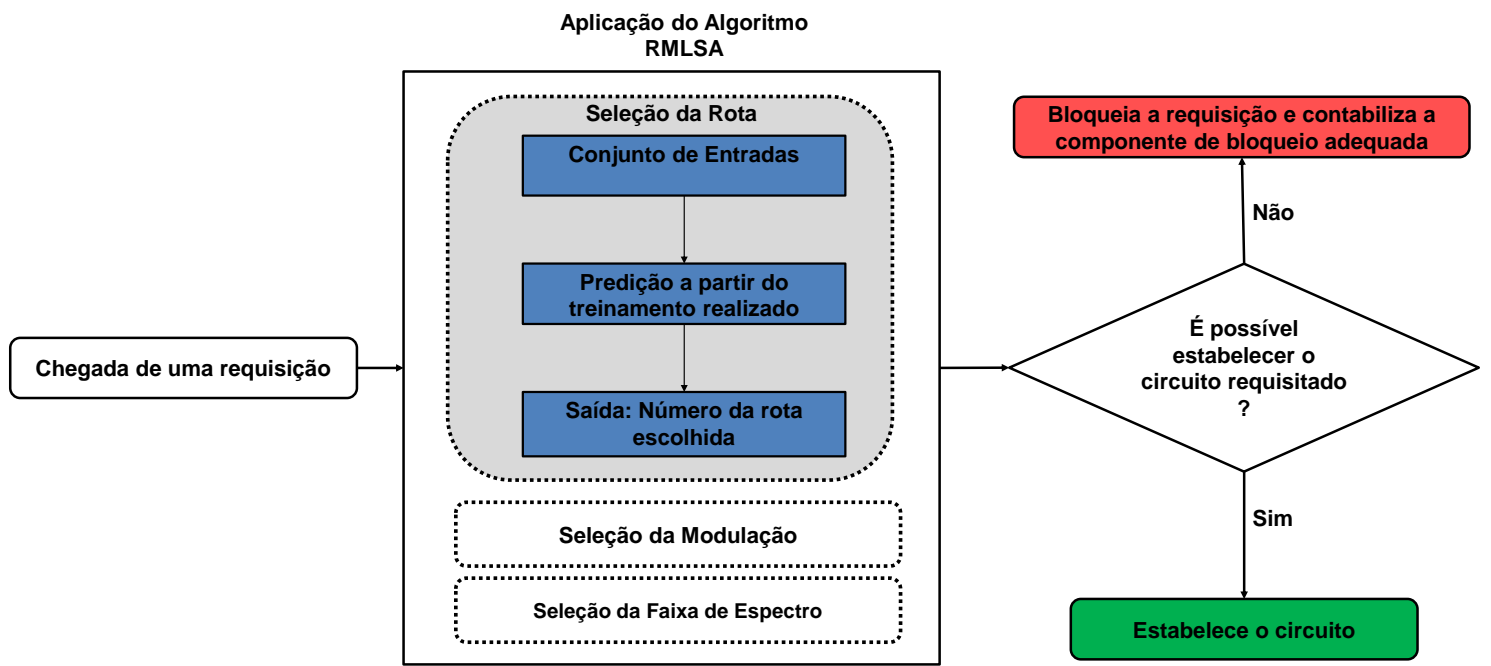

Figura 4. Fluxograma de aplicação do algoritmo IRON.

Conforme observado na Figura 4, logo após a chegada de uma requisição, é aplicado o algoritmo RMLSA. O primeiro passo é a escolha da rota, nessa etapa então é feito a coleta dos parâmetros que serão passados como entrada para a rede neural treinada (parâmetros de 'a' até 'h'), conforme Tabela 1. Após, a rede MLP treinada realiza então a predição, baseada nos novos valores de entrada e como resposta retorna o número da rota candidata a ser escolhida.

O roteamento utilizado neste trabalho é um roteamento do tipo fixo alternativo, ou seja, cada par de nós da rede mantém um lista com $k$ rotas candidatas fixas, computadas off-line. Quando é solicitado uma requisição, essas $k$ rotas são testadas em ordem até que uma delas satisfaça todos os requisitos para o estabelecimento do circuito.

Após a escolha da rota feita pela rede neural, é feita então uma reorganização na ordem de checagem das $k$ rotas candidatas. A rota escolhida pela rede neural passa a ser prioridade no momento da checagem, ou seja, passa a ser a primeira da lista das rotas candidatas. Isso foi feito para que o roteamento continuasse a ser do tipo fixo alternativo e caso não seja possível atender a requisição utilizando a rota escolhida pela rede neural, ainda seja possível realizar o atendimento com as outras duas rotas candidatas. Por exemplo, caso a rede neural tenha escolhido a rota número 3, a ordem de checagem passa a ser 3-1-2. Esse comportamento pode ser visualizado na Figura 5.

Por fim, o algoritmo RMLSA escolhe a modulação e uma faixa do espectro livre e apropriada para alocar o circuito óptico. A checagem é finalizada quando um primeiro conjunto de recursos (rota, modulação e espectro livre contíguo e contínuo) é encontrado em que seja possível estabelecer o circuito óptico. Após, o plano de controle verifica se é possível estabelecer o circuito óptico com os recursos definidos. Caso não seja possível 
Rota 1
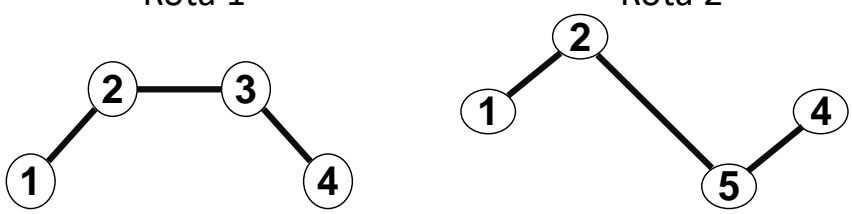

Rota 3

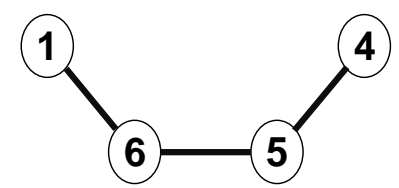

Ordem de checagem das rotas candidatas

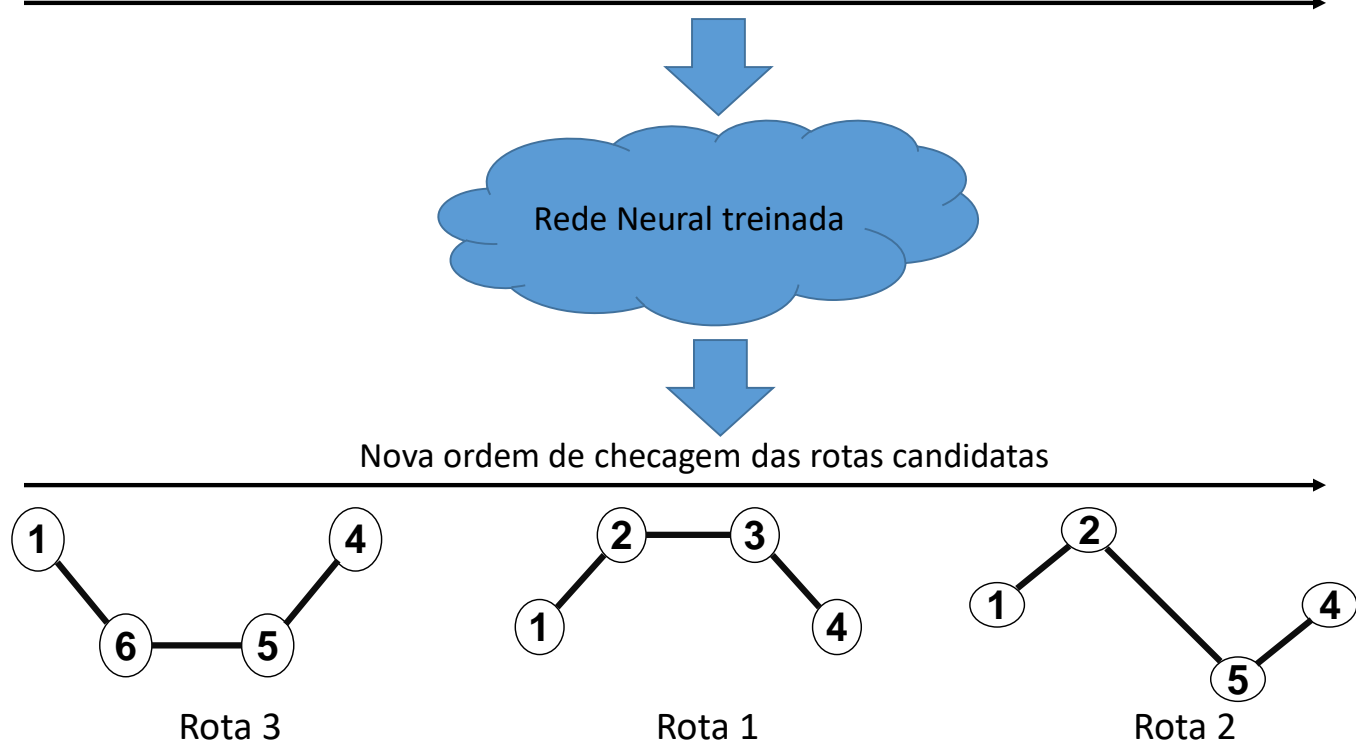

Figura 5. Comportamento do algoritmo IRON.

o estabelecimento deste novo circuito, é feito então a identificação e a contabilização do bloqueio em uma das componentes de bloqueio já mencionadas (Fragmentação, AEL, QoTN ou QoTO). Caso seja possível os recursos definidos anteriormente são alocados.

O IRON utiliza o First Fit para realizar a alocação espectral e para a seleção de modulação é utilizado o algoritmo de seleção do formato de modulação pela QoT, que escolhe a modulação $M$ mais eficiente espectralmente em que seja possível atender a requisição, respeitando as restrições de continuidade e contiguidade e respeitando o limiar de OSNR do circuito utilizando a rota $i$ e modulação $M$ [Fontinele et al. 2017].

\section{Avaliação de Desempenho}

A rede neural MLP foi implementada utilizando as bibliotecas de código aberto Tensorflow [Abadi et al. 2016] e Keras [Chollet et al. 2015] na linguagem de programação Python. O desempenho da rede durante o treinamento é medido através da métrica acurácia. Para calcular a acurácia é utilizado uma matriz de confusão, na qual a partir dela podemos calcular o Verdadeiro Positivo (VP), Falso Positivo (FP), Verdadeiro Negativo (VN) e Falso Negativo (FN) [Sokolova and Lapalme 2009]. A acurácia é calculada conforme Equação 1 a seguir.

$$
\text { Acuracia }=\frac{V P+V N}{V P+F P+V N+F N} .
$$

Podemos dividir o treinamento da rede em duas etapas utilizando o otimi- 
zador Adam [Kingma and Ba 2014], taxa de aprendizagem dinâmica e Early-Stop [Wang and Chen 2019]. Na primeira etapa a taxa de aprendizagem é igual a 0,001 até que o valor da perda tenha uma piora por 10 vezes seguidas. Após isso dá-se início a segunda etapa, na qual a taxa de aprendizagem é diminuída para 0,0001. O treinamento é então finalizado quando o valor de perda piora por 20 vezes consecutivas. Durante o treinamento, toda a base de dados foi utilizada, objetivando uma maior diversidade de amostras, visto que durante a simulação o cenário é dinâmico e novas amostras seriam produzidas, portanto, a base de treino é diferente da base de testes.

Considerando que existe uma quantidade limitada $k$ de rotas que podem ser escolhidas e que o objetivo da rede neural consiste em escolher uma delas, podemos dizer que trata-se de um problema de classificação. Dessa forma, a acurácia é utilizada para avaliar o treinamento da rede. A Tabela 2 abaixo apresenta a taxa de acurácia obtida com o treinamento, quantidade de registro de cada base de dados e o tempo de treinamento em segundos para as topologias Cost239 e Pan-European.

\section{Tabela 2. Acurácia, quantidade de registro e tempo de treinamento da rede neural para as topologias Cost239 e Pan-European.}

\begin{tabular}{c|c|c}
\hline & Cost239 & Pan-European \\
\hline Acurácia & $66,30 \%$ & $66,04 \%$ \\
\hline Quantidade registros & 841.975 & 899.697 \\
\hline Tempo de treinamento (s) & 847 & 2920 \\
\hline
\end{tabular}

A proposta IRON foi comparada com o algoritmo Complete Sharing. Ambos utilizando o valor de $k$ igual a 3, possuindo assim o mesmo número de rotas candidatas. A métrica probabilidade de bloqueio de banda foi utilizada como forma de avaliação. As componentes desse bloqueio também foram avaliadas: Fragmentação, QoTN (QoT inaceitável para um novo circuito), QoTO (QoT inaceitável para os circuitos já ativos na rede) e AEL (Ausência de Espectro Livre).

Todas as simulações foram realizadas no simulador SNetS (SLICE Network Simulator) [Fontinele et al. 2017]. Em cada simulação foram geradas 100.000 requisições de circuitos ópticos com sete níveis de granularidade: 100, 150, 200, 250, 300, 350 e 400 Gbps, com as proporções 7:6:5:4:3:2:1, respectivamente. A geração de requisições segue a distribuição de Poisson com a carga de tráfego distribuída uniformemente entre todos os pares de nós origem e destino. Para cada simulação foram realizadas dez replicações com diferentes sementes de geração de variável aleatória. Todos os resultados possuem nível de confiança de $95 \%$.

As topologias consideradas durante as simulações são a Cost239 e a PanEuropean, apresentadas na Figura 6. Foram considerados 5 formatos de modulações neste trabalho: BPSK, 4QAM, 8QAM, 16QAM e 32QAM e os seus respectivos limiares de OSNR por símbolo são 5,5, 8,5, 12,5, 15,1 e 18,1 dB [Ives et al. 2014]. Todos os enlaces da rede são bidirecionais e possuem largura de banda do espectro dividida em 320 slots de frequência. Um slot de frequência possui largura de banda de $12,5 \mathrm{GHz}$ e a banda de guarda utilizada foi de 1 slot. Os ganhos dos amplificadores são ajustados para compensar as perdas dos dispositivos (comutadores, multiplexadores e demultiplexadores) e das fibras. Outros parâmetros utilizados nas simulações estão listados na Tabela 3. 


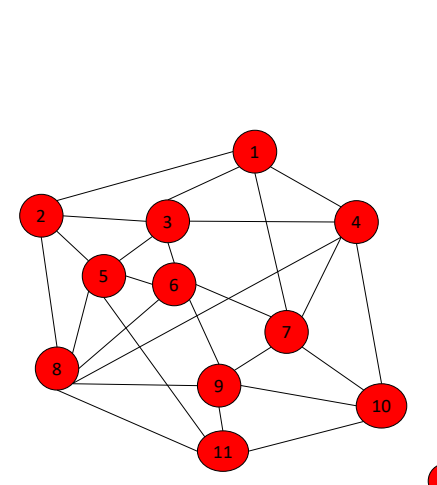

(a)

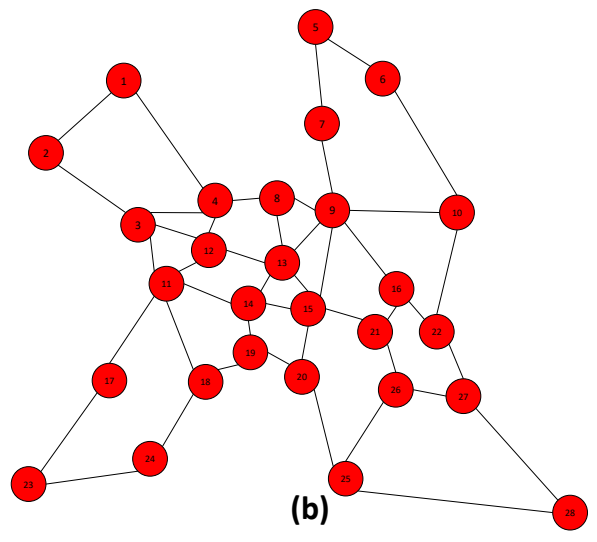

(b)

Figura 6. Topologias utilizadas: (a) Cost239 e (b) Pan-European

Tabela 3. Parâmetros de camada física utilizados nas simulações.

\begin{tabular}{c|c}
\hline Descrição & Valor \\
\hline Densidade espectral de potência do sinal & $-23 \mathrm{dBm} / \mathrm{GHz}$ \\
Atenuação da fibra $(\alpha)$ & $0,2 \mathrm{~dB} / \mathrm{km}$ \\
Parâmetro de dispersão da fibra $(D)$ & $16 \mathrm{ps} /(\mathrm{nm} \cdot \mathrm{km})$ \\
Coeficiente não linear da fibra $(\gamma)$ & $1,3(\mathrm{Wkm})^{-1}$ \\
Tamanho de um span $\left(L_{s}\right)$ & $80 \mathrm{~km}$ \\
Figura de ruído do amplificador $(N F)$ & $5 \mathrm{~dB}$ \\
\hline
\end{tabular}

A Figura 7 mostra a probabilidade de bloqueio de banda obtida pelos algoritmos Complete Sharing e IRON em função da carga da rede para as topologias Cost239 e Pan-European. Observa-se pela Figura 7 que o algoritmo IRON apresentou uma menor probabilidade de bloqueio para todos os pontos de carga em ambos os cenários.

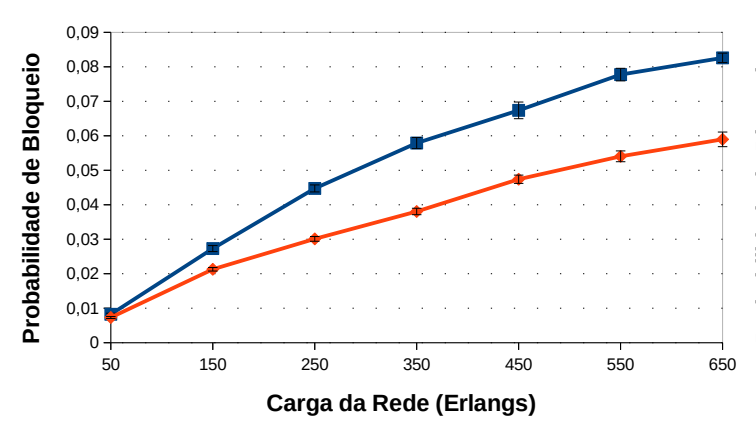

(a)

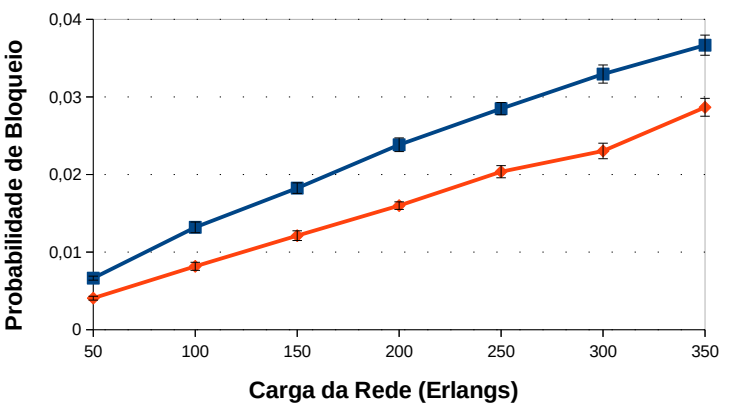

(b)

Figura 7. Probabilidade de bloqueio de banda para as topologias (a) Cost239 e (b) Pan-European.

Considerando o ponto de maior carga na topologia Cost239 (650 Erlangs), o algoritmo IRON obteve uma redução no bloqueio de banda de aproximadamente $28,59 \%$ em relação ao algoritmo Complete Sharing. Analisando a mesma situação para a topologia Pan-European, sob a carga de 350 Erlangs, o IRON obteve um bloqueio de aproximadamente $21,83 \%$ em relação ao Complete Sharing.

As componentes da probabilidade de bloqueio de banda obtidas com os algoritmos 
apresentados na Figura 7 são exibidas nas Tabelas 4 e 5 . Os resultados das Tabelas 4 e 5 foram obtidos ao analisar o último ponto de carga de cada topologia, 650 e 350 Erlangs para as topologias Cost239 e Pan-European respectivamente.

\section{Tabela 4. Componentes da probabilidade de bloqueio de banda para a topologia Cost239 na carga de 650 Erlangs.}

\begin{tabular}{c|c|c|c|c}
\hline Algoritmo & Fragmentação & QoTN & QoTO & AEL \\
\hline Complete Sharing & $0 \%$ & $0 \%$ & $100 \%$ & $0 \%$ \\
\hline IRON & $0,04 \%$ & $0 \%$ & $99,96 \%$ & $0 \%$ \\
\hline
\end{tabular}

Tabela 5. Componentes da probabilidade de bloqueio de banda para a topologia Pan-European na carga de 350 Erlangs.

\begin{tabular}{c|c|c|c|c}
\hline Algoritmo & Fragmentação & QoTN & QoTO & AEL \\
\hline Complete Sharing & $0 \%$ & $0 \%$ & $100 \%$ & $0 \%$ \\
\hline IRON & $7,34 \%$ & $0,02 \%$ & $92,50 \%$ & $0,14 \%$ \\
\hline
\end{tabular}

Observando as Tabelas 4 e 5, podemos verificar que a componente que mais impactou o bloqueio foi a QoTO. Vale ressaltar que o algoritmo Complete Sharing foi proposto para lidar com a fragmentação do espectro, reduzindo assim o bloqueio causado pelas componentes que não estão relacionadas a camada física.

O melhor desempenho do IRON está relacionado a habilidade das redes neurais de aprender e reconhecer padrões. Além disso, a priorização da rota escolhida pela rede neural mostra que por diversas vezes, nem sempre a menor rota pode ser a melhor opção. A partir das informações de todas as rotas candidatas, a rede neural pode inferir de maneira mais eficiente qual a melhor rota para um determinado estado da rede e requisição.

\section{Conclusão}

Este artigo propõe o algoritmo IRON para o problema de roteamento em redes ópticas elásticas. O IRON utiliza uma rede neural multi layer perceptron para selecionar a melhor rota, baseado no estado das $k$ rotas candidatas disponíveis.

O algoritmo IRON foi comparado com o algoritmo Complete Sharing, ambos utilizam um roteamento do tipo fixo-alternativo para escolha da rota. Os algoritmos foram comparados em dois cenários diferentes, utilizando as topologias Cost239 e PanEuropean. O algoritmo IRON obteve uma redução no bloqueio de banda de aproximadamente $28,59 \%$ e $21,83 \%$ em relação ao Complete Sharing, observando o último ponto de carga das topologias Cost239 e Pan-European respectivamente.

Neste artigo foi possível criar uma nova solução utilizando Machine Learning a partir de uma heurística já conhecida na literatura para formação de uma base de aprendizado contendo apenas os bons resultados dessa heurística. Dessa forma, a nova solução que utiliza ML consegue obter resultados melhores do que a estratégia original. Como trabalhos futuros, deseja-se estudar esse tipo de abordagem utilizando outras técnicas de ML em outros problemas encontrados nas redes ópticas elásticas. Pode-se ainda treinar e verificar o comportamento do algoritmo utilizando mais parâmetros, bem como utilizando um número $k$ maior de rotas. Além disso, pretende-se estudar melhorias na rede neural, de formar a aumentar a acurácia. 


\section{Referências}

Abadi, M., Barham, P., Chen, J., Chen, Z., Davis, A., Dean, J., Devin, M., Ghemawat, S., Irving, G., Isard, M., et al. (2016). Tensorflow: a system for large-scale machine learning. In $O S D I$, volume 16, pages 265-283.

Chatterjee, B. C., Sarma, N., and Oki, E. (2015). Routing and spectrum allocation in elastic optical networks: A tutorial. IEEE Communications Surveys \& Tutorials, 17(3):1776-1800.

Chollet, F. et al. (2015). Keras: Deep learning library for theano and tensorflow. URL: https://keras. io/k, 7(8).

Christodoulopoulos, K., Tomkos, I., and Varvarigos, E. (2011). Elastic bandwidth allocation in flexible OFDM-based optical networks. Journal of Lightwave Technology, 29(9):1354-1366.

Fontinele, A., Santos, I., Neto, J. N., Campelo, D. R., and Soares, A. (2017). An efficient IA-RMLSA algorithm for transparent elastic optical networks. Computer Networks, 118(Supplement C): 1 - 14.

Gardner, M. W. and Dorling, S. (1998). Artificial neural networks (the multilayer perceptron)-a review of applications in the atmospheric sciences. Atmospheric environment, 32(14-15):2627-2636.

Hawkins, D. M. (2004). The problem of overfitting. Journal of chemical information and computer sciences, 44(1):1-12.

Hecht-Nielsen, R. (1992). Theory of the backpropagation neural network. In Neural networks for perception, pages 65-93. Elsevier.

Ives, D. J., Bayvel, P., and Savory, S. J. (2014). Physical layer transmitter and routing optimization to maximize the traffic throughput of a nonlinear optical mesh network. In 2014 International Conference on Optical Network Design and Modeling, pages $168-173$.

Jinno, M., Takara, H., Kozicki, B., Tsukishima, Y., Sone, Y., and Matsuoka, S. (2009). Spectrum-efficient and scalable elastic optical path network: architecture, benefits, and enabling technologies. IEEE Communications Magazine, 47(11):66-73.

Johannisson, P. and Agrell, E. (2014). Modeling of nonlinear signal distortion in fiberoptic networks. Journal of Lightwave Technology, 32(23):4544-4552.

Khokhar, S., Zin, A. A. B. M., Mokhtar, A. S. B., and Pesaran, M. (2015). A comprehensive overview on signal processing and artificial intelligence techniques applications in classification of power quality disturbances. Renewable and Sustainable Energy Reviews, 51:1650-1663.

Kingma, D. P. and Ba, J. (2014). Adam: A method for stochastic optimization. arXiv preprint arXiv:1412.6980.

LeCun, Y., Bengio, Y., and Hinton, G. (2015). Deep learning. nature, 521(7553):436.

Levesque, M. and Elbiaze, H. (2009). Graphical probabilistic routing model for obs networks with realistic traffic scenario. In GLOBECOM 2009 - 2009 IEEE Global Telecommunications Conference, pages 1-6. 
Mohan, N., Soman, K., and Vinayakumar, R. (2017). Deep power: Deep learning architectures for power quality disturbances classification. In 2017 International Conference on Technological Advancements in Power and Energy (TAP Energy), pages 1-6. IEEE.

Rodrigues, W. L., Sousa Monteiro, N., Silva Borges, F. A., de Andrade Lira Rabelo, R., and Branco Soares, A. C. (2020). An adaptive guard band selection based on convolutional neural network. In 2020 IEEE International Conference on Systems, Man, and Cybernetics (SMC), pages 2814-2821.

Saradhi, C. V. and Subramaniam, S. (2009). Physical layer impairment aware routing (PLIAR) in WDM optical networks: Issues and challenges. Commun. Surveys Tuts., 11(4):109-130.

Sokolova, M. and Lapalme, G. (2009). A systematic analysis of performance measures for classification tasks. Information Processing \& Management, 45(4):427 - 437.

Trindade, S. and da Fonseca, N. L. S. (2021). Machine learning for spectrum defragmentation in space-division multiplexing elastic optical networks. IEEE Network, 35(1):326-332.

Troia, S., Rodriguez, A., Martín, I., Hernández, J. A., De Dios, O. G., Alvizu, R., Musumeci, F., and Maier, G. (2018). Machine-learning-assisted routing in sdn-based optical networks. In 2018 European Conference on Optical Communication (ECOC), pages $1-3$.

Wang, R. and Mukherjee, B. (2014). Spectrum management in heterogeneous bandwidth optical networks. Optical Switching and Networking, 11, Part A:83-91.

Wang, S. and Chen, H. (2019). A novel deep learning method for the classification of power quality disturbances using deep convolutional neural network. Applied Energy, 235:1126 - 1140 .

Wen Jin, Zhao Jia Li, Luo Si Wei, and Han Zhen (2000). The improvements of bp neural network learning algorithm. In WCC 2000 - ICSP 2000. 2000 5th International Conference on Signal Processing Proceedings. 16th World Computer Congress 2000, volume 3, pages 1647-1649 vol.3.

Wu, J., Ning, Z., and Guo, L. (2017). Energy-efficient survivable grooming in softwaredefined elastic optical networks. IEEE Access, 5:6454-6463.

Yan, L., Agrell, E., Wymeersch, H., Johannisson, P., Di Taranto, R., and Brandt-Pearce, M. (2015). Link-level resource allocation for flexible-grid nonlinear fiber-optic communication systems. IEEE Photonics Technology Letters, 27(12):1250-1253.

Zhao, J., Wymeersch, H., and Agrell, E. (2015). Nonlinear impairment aware resource allocation in elastic optical networks. In 2015 Optical Fiber Communications Conference and Exhibition (OFC), pages 1-3.

Zhong, Z., Hua, N., Yuan, Z., Li, Y., and Zheng, X. (2019). Routing without routing algorithms: An ai-based routing paradigm for multi-domain optical networks. In 2019 Optical Fiber Communications Conference and Exhibition (OFC), pages 1-3. 